\title{
Cognitive Aging and the Adaptive Use of Recognition in Decision Making
}

\author{
Thorsten Pachur \\ University of Basel
}

\author{
Rui Mata \\ University of Lisbon
}

\author{
Lael J. Schooler \\ Max Planck Institute for Human Development
}

\begin{abstract}
The recognition heuristic, which predicts that a recognized object scores higher on some criterion than an unrecognized one, is a simple inference strategy and thus an attractive mental tool for making inferences with limited cognitive resources-for instance, in old age. In spite of its simplicity, the recognition heuristic might be negatively affected in old age by too much knowledge, inaccurate memory, or deficits in its adaptive use. Across 2 studies, we investigated the impact of cognitive aging on the applicability, accuracy, and adaptive use of the recognition heuristic. Our results show that (a) young and old adults' recognition knowledge was an equally useful cue for making inferences about the world; (b) as with young adults, old adults adjusted their use of the recognition heuristic between environments with high and low recognition validities; and (c) old adults, however, showed constraints in their ability to adaptively suspend the recognition heuristic on specific items. Measures of fluid intelligence mediated these age-related constraints.
\end{abstract}

Keywords: aging, decision making, adaptive, recognition, memory

Supplemental materials: http://dx.doi.org/10.1037/a0017211.supp

Many decision-making-related abilities decline in old age (Chasseigne, Mullet, \& Stewart, 1997; Finucane, Mertz, Slovic, \& Schmidt, 2005; Hasher \& Zacks, 1988; Mutter, Strain, \& Plumlee, 2007). The aging decision maker seems to cope with these agerelated deficits adaptively - that is, by shifting to less demanding, but nonetheless effective, strategies (e.g., Chen \& Sun, 2003; Mata, Schooler, \& Rieskamp, 2007).

In this article, we examine age differences concerning the use of a particularly simple decision strategy, the recognition heuristic (Goldstein \& Gigerenzer, 2002). According to the recognition heuristic, if one of two objects is recognized but not the other, it is inferred that the recognized object scores higher on some criterion. For instance, having to judge which of two Dutch masters, Rembrandt or Frans Hals, has a higher market value, and provided one has heard of the former but not the latter, the heuristic predicts a Rembrandt to be more valuable. The heuristic exploits (a) a core human ability - recognition memory - and (b) the fact that the statistical structure of the environment giving rise to recognition (e.g., how often a painter is mentioned in the media) is often systematic. Moreover, the heuristic hinges on incomplete recogni-

Thorsten Pachur, Cognitive and Decision Sciences, Faculty of Psychology, University of Basel, Basel, Switzerland; Rui Mata, Department of Psychology, University of Lisbon, Lisbon, Portugal; Lael J. Schooler, Center for Adaptive Behavior and Cognition, Max Planck Institute for Human Development, Berlin, Germany.

We thank Shu-Chen Li and Yee-Lee Shing for many constructive comments and Laura Wiles for editing the article.

Correspondence concerning this article should be addressed to Thorsten Pachur, Cognitive and Decision Sciences, University of Basel, Missonsstrasse 60/62, 4055 Basel, Switzerland. E-mail: thorsten.pachur@unibas.ch tion knowledge, as it can only be applied when one of the two objects is unknown.

We address the following issues:

1. How do age-related changes in recognition memory affect the value of recognition for making inferences about the world? For illustration, assume that there is a correlation between whether one recognizes the name of a painter and how much one of his paintings would fetch at an auction. The strength of this correlation might well change with age as new artists are learned and old ones forgotten or when memory becomes less reliable.

2. How adaptive are young and old adults in using recognition to make inferences? We consider adaptivity on two levels. First, are young and old adults equally sensitive to the correlation between recognition and the criterion of interest in an environment? When the correlation between recognition and the criterion is high, it would be adaptive to rely heavily on the recognition heuristic; when the correlation is low, it would be adaptive to decrease reliance on the recognition heuristic. We refer to this global aspect of adaptivity as environment adaptivity. Second, are young and old adults equally able to suspend the use of the recognition heuristic when they have knowledge indicating that the heuristic would lead to an incorrect inference? We refer to this latter aspect of adaptivity as item adaptivity.

In the following, we first sketch the notion of the mind as an adaptive toolbox (e.g., Gigerenzer, Todd, \& the ABC Research 
Group, 1999; see also Payne, Bettman, \& Johnson, 1993) and how this notion can help us understand the aging decision maker. Second, we describe the recognition heuristic as one specific tool of the adaptive toolbox and summarize research on its adaptive use. Third, we discuss how possible changes in recognition memory in old age might impact the value of recognition as a valid cue for making inferences. Finally, we summarize our hypotheses about the effect of age-related cognitive change on the value of recognition as a cue and on the adaptive use of the recognition heuristic, and we describe two empirical studies that test these hypotheses.

\section{Adaptive Decision Making and Cognitive Aging}

Successful decision making requires the selection of strategies that match the specific characteristics of the current decision task as well as the resources available to the decision maker (Payne et al., 1993; see also Dunlosky \& Hertzog, 2000; Lemaire, Arnaud, \& Lecacheur, 2004). Gigerenzer et al. (1999; also see Gigerenzer \& Selten, 2001) proposed the metaphor of an adaptive toolbox to describe the notion that the decision maker is equipped with a repertoire of strategies and that adaptive decision making arises from selecting from this repertoire appropriately. Two strategies from the adaptive toolbox that have been well studied are the take-the-best heuristic (Gigerenzer \& Goldstein, 1996) and the recognition heuristic (Goldstein \& Gigerenzer, 2002). Both heuristics embody limited search and exploit the statistical structure of the environment. A number of studies have provided evidence that people select such simple strategies more when cognitive capacities are scarce (e.g., Bröder \& Schiffer, 2006; Pachur \& Hertwig, 2006; Rieskamp \& Hoffrage, 2008) or when the costs of information search are high (Bröder \& Schiffer, 2003).

How is the selection, and in particular, the adaptive selection of decision strategies affected by cognitive aging? To address this issue, Mata et al. (2007) asked young and old adults to infer which of two diamonds was more valuable, on the basis of various cues (e.g., size, clarity). There were two task environments. One environment was constructed such that the simple take-the-best heuristic (which makes a decision on the basis of only one cue) was appropriate; in the other environment, a more complex, weightedadditive strategy was more appropriate. As it turned out, both young and old adults tended to select the strategy appropriate for the respective environment. In spite of this adaptive strategy selection between environments, however, older adults showed a greater tendency to use simpler strategies. The age difference in strategy selection was mediated by individual differences in fluid abilities. Consequently, the results suggest that old adults, although adaptive, are constrained in their strategy selection by limited cognitive resources. However, do Mata et al.'s results generalize to the use of other strategies in the adaptive toolbox and, in particular, to situations when people can use knowledge they have learned outside, rather than inside the laboratory?

\section{The Recognition Heuristic and Its Adaptive Use}

A considerable amount of research conducted with young adults suggests that the recognition heuristic is often used (e.g., Frosch, Beaman, \& McCloy, 2007; Goldstein \& Gigerenzer, 2002; Pachur \& Biele, 2007; Pachur, Bröder, \& Marewski, 2008; Pohl, 2006;
Snook \& Cullen, 2006; but see Oppenheimer, 2003). Little is known, however, about its role in the decision making of old adults. Following Mata et al.'s (2007) study on the adaptive selection of the take-the-best heuristic, a key question is whether old adults use the recognition heuristic adaptively.

The degree to which recognition enables one to make a correct inference in an environment can be indexed by the recognition validity $\alpha$. It is calculated as

$$
\alpha=R /(R+W),
$$

where $R$ and $W$ equal the number of correct (right) and incorrect (wrong) inferences, respectively, that are made on those pairs in which one object is recognized and the other is not and the recognized object is judged to have the higher criterion value. If $\alpha=.5$, recognition is not correlated with the criterion; if $\alpha=1$, recognition is perfectly correlated with the criterion (i.e., it always leads to a correct inference).

Although recognition is a good cue in many real-world domains (e.g., cities' populations: Goldstein \& Gigerenzer, 2002; Pohl, 2006; success in sports: Pachur \& Biele, 2007; Scheibehenne \& Bröder, 2007; Snook \& Cullen, 2006; wealth: Frosch et al., 2007), it is not always. For instance, for predicting which of two French cities is larger, the recognition validity is .87 (on the basis of a sample of German participants; Goldstein \& Gigerenzer, 2002); however, it is only .60 for predicting which of two infectious diseases occurs more frequently in Germany (Pachur \& Hertwig, 2006). Consistent with the notion of an adaptive toolbox, Pachur, Todd, Gigerenzer, Schooler, and Goldstein (in press) found across 11 different environments a correlation of $r=.64$ between the average proportion with which (young) adults followed recognition and the average $\alpha$ in the respective environment. In other words, people seem to rely heavily on recognition when its validity is high and less so when its validity is low.

Using recognition adaptively not only requires adjusting reliance on it across environments but it requires selectively applying, or suspending, the recognition heuristic within an environment when item-specific knowledge indicates that it would lead to an incorrect decision (e.g., that cholera is practically extinct in Germany). Pachur and Hertwig (2006) argued that suspending the recognition heuristic requires cognitive resources because recognition has a retrieval primacy: Whereas searching for factual knowledge — which might indicate that following recognition yields an incorrect decision and thus leads to suspending the recognition heuristic-requires effortful retrieval, recognition knowledge is provided automatically. As a consequence, suspending the recognition heuristic is associated with higher cognitive costs than following it. To test their hypothesis, Pachur and Hertwig asked participants to judge which of two infectious diseases has a higher incidence rate in Germany-a domain with low recognition validity. As predicted, decisions that followed recognition were made faster than decisions that contradicted it (see also Volz et al., 2006). Suspension of the recognition heuristic occurred particularly frequently when participants had knowledge that conclusively contradicted recognition (e.g., that a recognized disease is practically extinct). Moreover, when cognitive resources were constrained by putting participants under time pressure (thus impeding the retrieval of knowledge), the proportion of decisions following recognition increased. Extending these results, Volz et al. (2006) observed in a functional magnetic resonance imaging 
study that suspending the recognition heuristic was associated with a deactivation within the anterior frontomedian cortex, an area that when active has been associated with being in a default mode of thinking (Gusnard, Akbudak, Shulman, \& Raichle, 2001). Going with the recognition heuristic may thus be the default, and going against it requires higher cognitive effort.

To summarize, the recognition heuristic, arguably the simplest strategy in the adaptive toolbox, exploits both recognition knowledge and the fact that recognition is often systematic and incomplete (i.e., we recognize the names of some Dutch masters but not all of them). Because recognition is provided by the cognitive system at less cognitive costs than factual knowledge, cognitive resources are necessary to suspend the use of the recognition heuristic-which is often appropriate in environments with low recognition validity. One interesting question, therefore, is to what degree age-related deficits in cognitive functioning might constrain the adaptive use of recognition when recognition is not a good cue. Another route by which aging might affect decision making is age-related change in recognition memory, which we turn to next.

\section{Age-Related Changes in Recognition Memory}

The recognition heuristic exploits a person's memory of previous encounters with an object in the real world. Pleskac (2007) has shown that deficits in recognition memory can hamper the heuristic's performance (but see Schooler \& Hertwig, 2005, showing that forgetting can also be beneficial for the heuristic). Although recognition is subject to age-related changes (albeit to a lesser degree than recall; e.g., Craik \& McDowd, 1987; La Voie \& Light, 1994), not all forms of recognition memory are equally affected. Consider the distinction between episodic and semantic recognition (cf. Neely \& Payne, 1983; Tulving, 1972). Episodic recognition hinges on the recollection of contextual information (such as source, time and place, feelings) about previous encounters with an object. It is usually measured by having participants study a list of known words and later asking them to discriminate these studied words from other known words that were not studied. Compared with young adults, old adults show considerable deficits in episodic memory tasks (e.g., Healy, Light, \& Chung, 2005; Kliegl \& Lindenberger, 1993; Spencer \& Raz, 1995). Semantic recognition, on the other hand, relies on context-independent features and thus does not require the recollection of episodic information. Importantly, semantic recognition seems to be rather robust across the lifespan (e.g., Allen et al., 2002).

The relative contribution of semantic and episodic recognition memory to the usefulness and to people's use of the recognition heuristic-and therefore the implication of cognitive aging on people's recognition knowledge-is currently unclear. To the extent that the recognition knowledge tapped by the recognition heuristic requires episodic memory, old adults' recognition knowledge might be a less valuable cue than young adults' recognition knowledge. Conversely, if recognition knowledge depends primarily on semantic recognition, with little contribution from episodic recognition, old and young adults' recognition knowledge might be similarly dependable for making inferences. By extension, it is conceivable that given old adults' greater experience, they might differ from young adults in terms of how often they can apply the recognition heuristic (as the applicability of the heuristic depends on how many objects are recognized). In the following, when we refer simply to recognition, we speak of semantic recognition. When referring to episodic recognition, we call it episodic recognition.

\section{Research Questions and Hypotheses}

Being able to make accurate decisions by relying on the recognition heuristic requires (a) that one's recognition knowledge discriminates between the objects and is valid and (b) that the recognition heuristic is applied adaptively. How might these requirements be affected in old age?

\section{Aging and Recognition Knowledge}

Recognition heuristic applicability. The applicability of the recognition heuristic depends on the number of objects that a person recognizes. If none or all of the objects are recognized, the heuristic is not applicable. There are two opposing possible scenarios concerning age differences in the number of recognized objects. On the one hand, old adults might recognize more objects than young adults because of their greater experience (and higher scores on measures of crystallized intelligence; e.g., Baltes, Staudinger, \& Lindenberger, 1999). On the other hand, it is possible that old adults recognize a similar number of or even fewer objects compared with young adults because of deficits in episodic recognition memory or the normal decaying of memory traces (cf. Schooler \& Hertwig, 2005).

Recognition validity. Age differences in recognition validity could arise because young and old adults might dwell in environments with different statistical structures (i.e., encounter different objects or do so with different frequencies). Moreover, on the basis of the simulations by Pleskac (2007), old adults' recognition validities might be lower if their recognition memory reflects the environment less accurately (e.g., when novel objects are falsely recognized). On the other hand, the simulation by Schooler and Hertwig (2005) showed that forgetting can also lead to higher recognition validities. On the basis of these results, the recognition validity of old adults might also be higher than those of young adults. In light of the examples of maintained semantic recognition memory (e.g., Allen et al., 2002), however, it is possible that changes in recognition validity occur only when memory is severely disrupted.

\section{Aging and the Adaptive Use of Recognition: Reduced Item Adaptivity Hypothesis}

We expected both young and old adults to show environment adaptivity by adjusting their reliance on recognition between environments that have high and low recognition validities. However, we expected old adults to show reduced item adaptivity, that is, to show a lower ability (compared with young adults) to go against recognition on items in which it would be beneficial to do so (which is key in particular in environments in which the recognition validity is low).

This latter hypothesis is motivated by (a) Pachur and Hertwig's (2006) finding that additional cognitive resources are required to search for factual knowledge (which might overturn recognition) and (b) the tendency of cognitive resources to be reduced in old 
age (e.g., Li et al., 2004). As a corollary of this hypothesis, we predict that old adults' reduced item adaptivity in using the recognition heuristic is mediated by age differences in the availability of cognitive resources - as measured by fluid components of cognitive functioning, such as speed-needed to retrieve the information necessary to suspend the use of the recognition heuristic.

\section{Study 1}

The main goal of this study was to compare old and young adults' (a) recognition knowledge and validity as well as (b) adaptive use of recognition when making probabilistic inferences in real-world domains. Participants judged either which of two infectious diseases has a higher annual incidence rate in Germany-an environment with low recognition validity (Pachur \& Hertwig, 2006) — or which of two American cities has more inhabitants - an environment with a high recognition validity (Volz et al., 2006). We also asked participants to indicate which of the diseases and cities, respectively, they had heard of before the experiment; and we measured participants' cognitive capacities on tests for verbal knowledge, speed, and episodic recognition memory.

\section{Method}

Participants. A total of 40 young adults ( 24 women, 16 men; mean age $=25.3$ years, range $=19-33$ years $)$ and 40 old adults
( 15 women, 25 men; mean age $=71$ years, range $=65-86$ years $)$ participated in this study, which was conducted at the Max Planck Institute for Human Development (Berlin, Germany). Young and old adults had, on average, 17.1 and 14.4 years of education, respectively. To deal with these differences in educational achievement, in all critical analyses we included years of education as a covariate (also in Study 2). Overall, young adults took approximately $120 \mathrm{~min}$ and old adults took approximately $150 \mathrm{~min}$ to complete all tasks. Participants received a fixed hourly payment of 10 euros as well as an extra bonus dependent on their performance (see below).

Tasks and materials. In the inference task, participants were presented with pairs of names of infectious diseases (or American cities) and asked to pick the disease with the higher annual incidence rate in a typical year in Germany (or city with the larger population). Previous analyses revealed recognition to have a low validity in the disease environment (Pachur \& Hertwig, 2006) and a high validity in the city environment (Volz et al., 2006). In the recognition task, they indicated which of the infectious diseases (or cities) they recognized. The two environments consisted of the names of the 24 notifiable infectious diseases used in Pachur and Hertwig (2006; see also Hertwig, Pachur, \& Kurzenhäuser, 2005) and the names of the 24 American cities used in Volz et al. (2006; see Table 1). To equate the applicability of the recognition heuristic in the disease and city environments, we selected the American cities such that their recognition rates (on the basis of the data

Table 1

The 24 Diseases and Cities Used As Target Objects in Studies 1 and 2

\begin{tabular}{|c|c|c|c|c|c|c|c|c|c|}
\hline \multirow[b]{5}{*}{ Target object } & \multirow{2}{*}{\multicolumn{5}{|c|}{ Infectious diseases }} & \multicolumn{4}{|c|}{ American cities } \\
\hline & & & & & & \multirow[b]{4}{*}{ Target object } & \multirow[b]{4}{*}{ Population } & \multirow{3}{*}{\multicolumn{2}{|c|}{$\begin{array}{l}\begin{array}{c}\text { Recognized by } \% \\
\text { of participants }\end{array} \\
\text { Study } 1(n=40)\end{array}$}} \\
\hline & \multirow{3}{*}{$\begin{array}{c}\text { Annual } \\
\text { incidence rate }\end{array}$} & \multicolumn{4}{|c|}{ Recognized by $\%$ of participants } & & & & \\
\hline & & \multicolumn{2}{|c|}{ Study $1(n=40)$} & \multicolumn{2}{|c|}{ Study $2(n=120)$} & & & & \\
\hline & & Young & Old & Young & Old & & & Young & Old \\
\hline Poliomyelitis & 0.25 & 100 & 100 & 98 & 97 & Grand Rapids & 199,700 & 25 & 25 \\
\hline Diphtheria & 1 & 90 & 100 & 98 & 100 & Spokane & 200,800 & 15 & 10 \\
\hline Trachoma & 1.75 & 15 & 5 & 13 & 30 & Garland & 224,000 & 5 & 20 \\
\hline Tularemia & 2 & 5 & 10 & 2 & 5 & Baton Rouge & 229,700 & 25 & 20 \\
\hline Cholera & 3 & 100 & 100 & 100 & 100 & Lexington-Fayette & 264,700 & 10 & 10 \\
\hline Leprosy & 5 & 100 & 100 & 98 & 100 & Raleigh & 285,800 & 30 & 10 \\
\hline Tetanus & 9 & 100 & 95 & 100 & 100 & Santa Ana & 348,100 & 65 & 45 \\
\hline Hemorrhagic fever & 10 & 45 & 30 & 27 & 42 & Minneapolis & 392,100 & 100 & 100 \\
\hline Botulism & 15 & 25 & 60 & 7 & 62 & Tulsa & 399,100 & 25 & 35 \\
\hline Trichinosis & 22 & 15 & 55 & 10 & 72 & Mesa & 418,800 & 10 & 20 \\
\hline Brucellosis & 23 & 5 & 35 & 8 & 43 & Fresno & 440,400 & 30 & 25 \\
\hline Leptospirosis & 39 & 20 & 5 & 8 & 15 & Fort Worth & 555,200 & 25 & 30 \\
\hline Gas gangrene & 98 & 30 & 55 & 15 & 75 & Denver & 583,000 & 95 & 100 \\
\hline Ornithosis & 119 & 15 & 5 & 3 & 25 & Nashville & 583,700 & 95 & 100 \\
\hline Typhoid and paratyphoid & 152 & 100 & 95 & 97 & 100 & El Paso & 585,300 & 75 & 80 \\
\hline Q fever & 179 & 15 & 20 & 3 & 23 & Detroit & 960,300 & 100 & 100 \\
\hline Malaria & 936 & 100 & 100 & 100 & 100 & Dallas & $1,234,200$ & 100 & 100 \\
\hline Syphilis & 1,514 & 100 & 100 & 98 & 100 & San Diego & $1,260,000$ & 100 & 100 \\
\hline Shigellosis & 1,627 & 10 & 5 & 7 & 10 & Phoenix & $1,395,800$ & 95 & 95 \\
\hline Gonorrhea & 2,926 & 85 & 100 & 95 & 100 & Philadelphia & $1,522,900$ & 100 & 100 \\
\hline Meningitis and encephalitis & 4,019 & 100 & 90 & 92 & 100 & Houston & $2,028,500$ & 100 & 100 \\
\hline Tuberculosis & 12,619 & 100 & 100 & 100 & 100 & Chicago & $2,934,900$ & 100 & 100 \\
\hline Viral hepatitis & 14,889 & 95 & 95 & 85 & 98 & Los Angeles & $3,805,400$ & 100 & 100 \\
\hline Gastroenteritis & 203,864 & 100 & 100 & 100 & 100 & New York & $8,103,700$ & 100 & 95 \\
\hline
\end{tabular}


collected by Volz et al., 2006) roughly matched those of the infectious diseases. The inference task consisted of all 276 possible pair comparisons of the 24 diseases (or cities). The recognition task comprised all 24 diseases (or cities). We assessed participants' cognitive capacities using a battery of psychometric tests that included tests of verbal knowledge (spot a word; Lindenberger, Mayr, \& Kliegl, 1993), processing speed (digit symbol substitution; Lindenberger et al., 1993), and episodic recognition memory (Shing, Werkle-Bergner, Li, \& Lindenberger, 2008). The episodic recognition memory tasks included both an item recognition task and an associative recognition task (e.g., Healy et al., 2005; Kelley \& Wixted, 2001; Naveh-Benjamin, 2000; Yonelinas, 1997). A detailed description of each test can be found in the online supplemental materials.

Design. The order of the inference and recognition tasks was counterbalanced. Overall, the design was thus a 2 (age group: young vs. old) $\times 2$ (environment: low vs. high recognition validity) $\times 2$ (task order: the recognition task preceded or followed the inference task) between-subjects design. Participants in each age group were randomly assigned to one of the four conditions resulting from crossing the task order and environment factors.

Procedure. After reading an introductory text (see the online supplemental materials for details), participants were first presented with either the inference task or the recognition task (depending on the condition). In the inference task, pairs of the names of diseases (or cities) were displayed on the left and right side of a computer screen (ordered randomly), and participants were asked to indicate their inference by pressing one of two keys. Response times were recorded. Participants were told that they would earn four cents for each correct inference and lose four cents for each wrong one (but no feedback was given during the task). Following a short practice phase, the 276 pairs were presented in 12 blocks (each containing 23 pairs), after each of which participants could take a short break. The pairs of objects were presented in a different random sequence to each participant within an age group, but groups were matched regarding the sequences (yoked design). In the recognition task, participants saw the names of the 24 diseases (or cities) one at a time (in a random order) on the computer screen and were asked to indicate whether they had heard of the disease (or city) before the experiment by pressing one of two keys. After the conclusion of the inference and recognition tasks, participants were administered the battery consisting of tests of verbal knowledge, speed, and episodic recognition memory. After each task, participants were given the opportunity to rest for a while if necessary.

\section{Results}

All analyses of variance included the factors of age group, environment, and task order, as well as years of education as a covariate.

Accuracy in the inference task. Table 2 shows that in both environments, old adults achieved a slightly higher overall accuracy than young adults, but the effects were small $(M \mathrm{~s}=71.9$ vs. $70.4), F(1,76)=1.45, p=.23$. There were no age differences in participants' accuracy on the subset of items in which both diseases were recognized (and where the recognition heuristic was thus not applicable)—which Goldstein and Gigerenzer (2002) called knowledge validity- $F(1,71)=0.14, p=.71$. Knowledge validity is assumed to reflect the amount of relevant factual knowl-

Table 2

Performance in the Inference Task and Results for the Recognition Heuristic (RH)

\begin{tabular}{|c|c|c|c|c|c|c|}
\hline \multirow[b]{3}{*}{ Measure/age group } & \multicolumn{4}{|c|}{ Study 1} & \multirow{2}{*}{\multicolumn{2}{|c|}{$\begin{array}{c}\text { Study } 2 \\
\begin{array}{c}\text { Diseases (low } \\
\text { recognition validity) }\end{array}\end{array}$}} \\
\hline & \multicolumn{2}{|c|}{$\begin{array}{l}\text { Cities (high } \\
\text { recognition validity) }\end{array}$} & \multicolumn{2}{|c|}{$\begin{array}{l}\text { Diseases (low } \\
\text { recognition validity) }\end{array}$} & & \\
\hline & $M$ & $S D$ & $M$ & $S D$ & $M$ & $S D$ \\
\hline \multicolumn{7}{|c|}{$\%$ of accurate judgments in the inference task } \\
\hline Young & 78.0 & 4.8 & 62.8 & 6.8 & 62.8 & 4.8 \\
\hline Old & 80.5 & 4.2 & 63.3 & 5.4 & 64.8 & 6.1 \\
\hline \multicolumn{7}{|l|}{ Knowledge validity } \\
\hline Young & 0.76 & 0.07 & 0.70 & 0.08 & 0.69 & 0.07 \\
\hline Old & 0.76 & 0.07 & 0.69 & 0.08 & 0.70 & 0.07 \\
\hline \multicolumn{7}{|l|}{ No. of recognized objects } \\
\hline Young & 15.3 & 3.1 & 14.7 & 2.3 & 13.7 & 1.4 \\
\hline Old & 15.2 & 3.1 & 15.6 & 2.3 & 17.0 & 2.4 \\
\hline \multicolumn{7}{|l|}{ Recognition validity } \\
\hline Young & 0.90 & 0.05 & 0.62 & 0.08 & 0.60 & 0.05 \\
\hline Old & 0.92 & 0.07 & 0.60 & 0.04 & 0.59 & 0.06 \\
\hline \multicolumn{7}{|l|}{$\%$ of inferences in line with RH } \\
\hline Young & 95.2 & 6.7 & 63.6 & 18.2 & 68.3 & 15.7 \\
\hline Old & 96.0 & 4.2 & 71.4 & 15.7 & 70.9 & 13.0 \\
\hline \multicolumn{7}{|l|}{ Item adaptivity $\left(d_{R H}^{\prime}\right)$} \\
\hline Young & 0.53 & 0.57 & 0.60 & 0.40 & 0.69 & 0.34 \\
\hline Old & 1.1 & 0.58 & 0.49 & 0.34 & 0.58 & 0.38 \\
\hline \multicolumn{7}{|l|}{ Environment adaptivity $\left(c_{R H}\right)$} \\
\hline Young & -1.61 & 0.43 & -0.32 & 0.56 & -0.49 & 0.52 \\
\hline Old & -1.41 & 0.50 & -0.59 & 0.53 & -0.56 & 0.44 \\
\hline
\end{tabular}

Note. Values of $c_{R H}<0$ indicate a tendency to rely on recognition in the inference task. The more negative the value, the stronger the reliance. 
edge that a person has about the respective environment and is calculated as the proportion of correct inferences on pairs in which both objects are recognized.

Recognition knowledge. As can be seen in Table 2, young and old adults did not differ in terms of the number of objects that they indicated having heard of before the experiment $(M \mathrm{~s}=15.0 \mathrm{vs}$. $15.4), F(1,76)=0.49, p=.49$. There was no interaction between environment and age group, $F(1,76)=0.61, p=.44$. The proportion of pair comparisons in the inference task in which young and old adults recognized one object but not the other-thus making the recognition heuristic applicable-was .46 and .45 , respectively. The number of recognized objects was unaffected by whether the recognition task followed the inference task or preceded it $(M \mathrm{~s}=15.5$ vs. 14.9), $F(1,76)=1.1, p=.30$ (cf. Pachur et al., 2008; Pachur \& Hertwig, 2006; Pohl, 2006). This was the case for both age groups, that is, there was no interaction between age group and task order, $F(1,76)=0.91, p=.34$.

Next we calculated, for each participant, the recognition validity on the basis of Equation 1. As intended, the recognition validity was considerably higher in the city environment than in the disease environment, $F(1,76)=462.7, p=.001$ (see Table 2). More importantly, the recognition validities did not differ between the age groups, $F(1,76)=0.13, p=.72$. The interaction between environment and age group was not significant, $F(1,76)=2.0$, $p=.16$. Table 1 shows, separately for young and old adults, for each disease and each city its recognition rate (i.e., the proportion of participants recognizing it). In both environments, the recognition rates were highly correlated between young and old adultsdiseases: $r=.93, p=.001$; cities: $r=.98, p=.001$-and did not differ in absolute terms- diseases: $t(23)=-1.2, p=.24$; cities: $t(23)=0.13, p=.90$.

To summarize, we find no evidence that the amount and pattern of recognized objects, or the validity of recognition knowledge, differed between young and old adults. That is, recognition was a similarly useful cue for making inferences for the two age groups. Next, we turn to the question of whether younger and older adults also relied on recognition in similar ways.

Environment adaptivity and item adaptivity. As expected, the percentage of inferences following recognition (i.e., the proportion of inferences in which participants judged the recognized object to have a higher criterion value) was considerably higher in the environment with a high recognition validity than in the environment with a low recognition validity $(M \mathrm{~s}=95.6 \%$ vs. $67.5 \%)$, $F(1,75)=96.4, p=.001$. Old adults followed recognition more often in the low recognition validity environment than young adults $(M \mathrm{~s}=71.4 \%$ vs. $63.6 \%$; see Table 2$)$, whereas in the high recognition validity environment, the percentages of the age groups were very similar $(M \mathrm{~s}=96.0 \%$ vs. $95.2 \%)$. The interaction between age group and environment was not significant, $F(1$, $75)=1.55, p=.22$-possibly because of the small sample size. Both young and old adults thus showed environment adaptivity. ${ }^{1}$

Supporting the notion that recognition has a retrieval primacy (which might constrain its adaptive use), Figure 1 shows that participants' inferences were considerably faster when they followed recognition compared with when they went against it (cf. Pachur \& Hertwig, 2006; Volz et al., 2006). Although old adults were generally slower at responding in the inference task, they showed the same pattern as young adults.
According to the reduced item adaptivity hypothesis, old adults' limited cognitive resources constrain their ability to discriminate individual cases in which the recognition heuristic arrives at correct and incorrect inferences. This constraint should manifest itself most clearly in the low recognition validity environment because there people often have to suspend the recognition heuristic to make a correct inference. To measure a person's ability to use recognition adaptively, we analyzed participants' inferences using signal detection theory (SDT). In this analysis, a person's item adaptivity was measured by the parameter $d_{R H}^{\prime}$, which reflects a person's ability to discriminate between cases in which recognition leads to correct inferences from those in which it leads to incorrect ones (cf. Pachur \& Hertwig, 2006; see the Appendix A for further details; for a similar approach, see Hilbig \& Pohl, 2008). The higher $d_{R H}^{\prime}$, the better the person is able to selectively suspend the recognition heuristic when it leads to an incorrect inference.

Unexpectedly, collapsed across both environments, old adults showed higher $d_{R H}^{\prime}$ than the young adults $(M \mathrm{~s}=0.79$ vs. 0.57$)$, $F(1,71)=4.9, p=.03$. Crucially, however, there was an interaction between age group and environment, $F(1,71)=9.6, p=$ .003. Whereas in the city environment old adults had a higher $d_{R H}^{\prime}$ than young adults $(M \mathrm{~s}=1.1$ vs. 0.53$), t(38)=3.1, p=.004$, in the disease environment, old adults showed a lower $d_{R H}^{\prime}(M \mathrm{~s}=$ 0.49 vs. 0.60$), t(38)=0.92, p=.34$. The latter result is consistent with the reduced item adaptivity hypothesis, but the reversal of the age differences between the two environments was not predicted. Consistent with the notion that $d_{R H}^{\prime}$ was predominantly driven by difficulties in suspending the recognition heuristic, the false alarm rate (i.e., the probability of erroneously going with recognition) was a stronger predictor of $d_{R H}^{\prime}$ than the hit rate (i.e., the probability of correctly going with recognition)_diseases: $r \mathrm{~s}=-.49$ versus .12; cities: $r \mathrm{~s}=-.46$ versus .43 . The order of the inference and recognition tasks had no effect on $d_{R H}^{\prime}, F(1,71)=1.1, p=$ .30 , and there was not an interaction between task order and age group, $F(1,71)=0.65, p=.42$.

In addition to $d_{R H}^{\prime}$, we calculated people's tendency to rely on recognition using the SDT parameter $c_{R H}$ (cf. Banks, 1970; Macmillan \& Creelman, 1990), which can be viewed as another measure of environment adaptivity. ${ }^{2}$ Values of $c_{R H}<0$ indicate a

\footnotetext{
${ }^{1}$ Although the task order of the recognition and the inference tasks did not affect the overall tendency to rely on recognition (as measured by $c_{R H}$ ), $F(1,71)=0.992, p=.32$, there was a significant interaction between task order and age group, $F(1,71)=5.2, p=.03$. Specifically, young adults decreased their reliance on recognition (in the inference task) when they had been presented with the names of the diseases in the recognition task $(M \mathrm{~s}=-1.04$ vs. -0.90$)$, whereas old adults increased their reliance $(M \mathrm{~s}=-0.82$ vs. -1.18$)$. One possible explanation for this result is that young and old adults react differently to the potentially priming effect of the recognition task. Specifically, young adults may have been aware of the potentially priming effect of the recognition task and (over)corrected for it, whereas the recognition task primed old adults to use recognition as a cue in the inference task (cf. Hess, McGee, Woodburn, \& Bolstad, 1998; Oppenheimer, 2004; Toth \& Daniels, 2002).

${ }^{2}$ We measured participants' tendency to rely on recognition using $c$ rather than $L$ (for an overview, see Stanislaw \& Todorov, 1999) because the latter showed some abnormalities in cases in which a person's false alarm rate was larger than his or her hit rate (for a definition of false alarm and hits rates, see the Appendix A). The $c$ index proved to be more robust in these cases.
} 


\section{Infections}

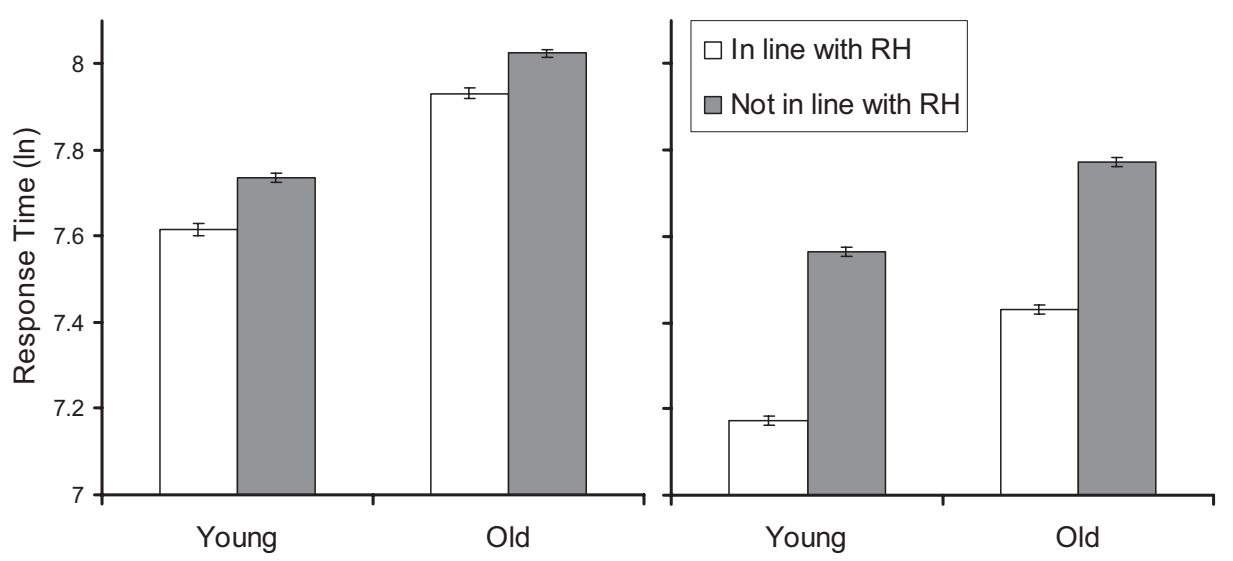

Figure 1. Log-transformed response times (RTs) of inference in which the recognition heuristic (RH) was applicable as a function of whether the inference was in line with the RH (RTs were log-transformed to correct the skewness of RT distributions). Error bars indicate standard errors of the mean. With regard to the untransformed data, in the disease and city environments, young adults' median RTs when their inference followed recognition were $1,856.5$ and $1,180.5 \mathrm{~ms}$, respectively, whereas they were 2,094 and $1,735 \mathrm{~ms}$, respectively, when the inference contradicted recognition. For old adults, in the disease and city environments, the median RTs were 2,644 and 1,553 ms, respectively, when their inference followed recognition, and 2,931 and 2,246.5 ms, respectively, when the inference contradicted recognition.

tendency to rely on recognition; $c_{R H}=0$ indicates that there is no tendency either to follow or to go against recognition. For both age groups, the average $c_{R H}$ was negative (see Table 2). In addition, $c_{R H}$ differed between the low and high validity environments in an appropriate fashion, with a more conservative reliance on recognition in the low validity than in the high validity environments $(M \mathrm{~s}=-0.46$ vs. -1.51$), F(1,71)=97.8, p=.001$. Interestingly, the difference in decision tendencies between the two environments was larger for young than for old adults, $F(1,71)=4.8, p=$ .03 (see Table 2). One explanation could be that because of larger unsystematic variance in old adults' decisions (cf. Ratcliff, Thapar, \& McKoon, 2007; Sanford \& Maule, 1971), their decision behavior is less extreme.

Cognitive capacity measures. Table 3 shows that, replicating previous findings (Baltes et al., 1999), old adults achieved higher scores than young adults on verbal knowledge but lower scores on both cognitive speed and the two measures of episodic recognition memory (cf. Naveh-Benjamin, 2000). The correlations among the different cognitive capacity measures are shown in Table 4. Next, we examine to what degree these age differences in cognitive capacity were associated with the observed age differences for the recognition heuristic.

Cognitive capacity and the recognition heuristic. From the cognitive capacity measures, only verbal knowledge was correlated with the number of recognized objects $(r=.20, p=.07)$. A corollary of the reduced item adaptivity hypothesis was that old adults' lower item adaptivity should be mediated by cognitive speed. To test this hypothesis, we conducted a mediation analysis (Baron \& Kenny, 1986; Judd \& Kenny, 1981) for the data in the disease environment (we focus on the low recognition validity environment because here the ability to suspend the recognition heuristic is key). In a first step, we regressed item adaptivity (as measured by $\left.d_{R H}^{\prime}\right)$ on age, yielding a negative association $(\beta=$ $-.16, p=.34)$. In a second step, we regressed cognitive speed on age, yielding a strongly negative association $(\beta=-.73, p=.001)$. In a third step, we regressed $d_{R H}^{\prime}$ on cognitive speed (holding age constant), yielding a positive association $(\beta=.22, p=.37)$. In addition, there was a positive association between $d_{R H}^{\prime}$ and speed within both age groups, with $r=.14$ for both the young and old adults. Finally, when we regressed $d_{R H}^{\prime}$ on age while holding cognitive speed constant, the relationship between $d_{R H}^{\prime}$ and age disappeared ( $\beta=.001, p=.99$ ), fulfilling the conditions required to establish mediation (Baron \& Kenny, 1986). Thus, as hypothesized, age differences in cognitive speed seem to mediate the age differences in the how adaptively recognition is used on an itemby-item basis. Nevertheless, Sobel's (1982) test for mediation was not significant $(z=-0.90, p=.82$; but note that Sobel's test is very conservative; MacKinnon, Warsi, \& Dwyer, 1995). Mediation analyses for the high recognition validity environmentreported in the Appendix B-indicated that neither speed, nor verbal knowledge, nor knowledge validity mediated the age differences in item adaptivity in this environment.

\section{Discussion of Study 1}

Study 1 suggests that - in spite of age-related deficits in episodic recognition memory - the recognition knowledge that people acquired in natural environments is robust in old age and enables the aging decision maker to make similarly good decisions as those made by young adults. In addition, evidence was provided that, like young adults, old adults relied considerably less on recognition in an environment in which the recognition validity was low compared with one 
Table 3

Young and Old Adults' Performance on the Battery of Cognitive Capacity Tests

\begin{tabular}{|c|c|c|c|c|c|c|c|c|c|c|c|c|}
\hline \multirow[b]{3}{*}{ Measure } & \multicolumn{6}{|c|}{ Study 1} & \multicolumn{6}{|c|}{ Study 2} \\
\hline & \multicolumn{2}{|c|}{ Young adults } & \multicolumn{2}{|c|}{ Old adults } & \multicolumn{2}{|c|}{ Statistical test } & \multicolumn{2}{|c|}{ Young adults } & \multicolumn{2}{|c|}{ Old adults } & \multicolumn{2}{|c|}{ Statistical test } \\
\hline & $M$ & $S D$ & $M$ & $S D$ & $t$ & $p$ & $M$ & $S D$ & $M$ & $S D$ & $t$ & $p$ \\
\hline $\begin{array}{l}\text { Verbal knowledge } \\
\text { Speed }\end{array}$ & 22.5 & 5.1 & 27.2 & 5.0 & 4.1 & .001 & 22.4 & 4.9 & 28.7 & 4.0 & 7.8 & .001 \\
\hline Composite & - & - & - & - & - & - & 0.77 & 0.70 & -0.77 & 0.58 & 13.0 & .001 \\
\hline $\begin{array}{l}\text { Identical pictures } \\
\text { Digit symbol }\end{array}$ & 38.5 & 4.65 & 26.8 & 4.51 & 11.5 & .001 & 39.3 & 4.44 & 28.1 & 3.90 & 14.7 & .001 \\
\hline $\begin{array}{l}\text { substitution } \\
\text { Inhibition }\end{array}$ & - & - & - & - & - & - & 58.6 & 11.1 & 44.8 & 8.4 & 7.7 & .001 \\
\hline Composite & - & - & - & - & - & - & 0.04 & 0.76 & -0.04 & 1.20 & 0.43 & .67 \\
\hline Flanker & - & - & - & - & - & - & -10.0 & 26.1 & -15.7 & 32.8 & 1.1 & .29 \\
\hline $\begin{array}{l}\text { Stroop } \\
\text { Episodic recognition } \\
\text { memory }\end{array}$ & - & - & - & - & - & - & -19.1 & 46.6 & -150.3 & 135.3 & 7.1 & .001 \\
\hline$d_{I T E M}^{\prime}$ & 2.46 & 0.79 & 1.84 & 0.78 & 3.5 & .001 & 2.35 & 0.74 & 1.78 & 0.67 & 4.4 & .001 \\
\hline$d_{A S S O C}$ & 2.25 & 1.07 & 0.99 & 0.83 & 5.9 & .001 & 2.10 & 1.10 & 0.89 & 0.86 & 6.7 & .001 \\
\hline$L_{I T E M}$ & 2.49 & 1.95 & 1.64 & 1.58 & 2.1 & .04 & 2.21 & 1.74 & 2.29 & 1.93 & -0.25 & .80 \\
\hline$L_{A S S O C}$ & 1.72 & 1.28 & 1.08 & 0.45 & 3.0 & .004 & 2.03 & 1.48 & 1.08 & 0.31 & 4.9 & .001 \\
\hline
\end{tabular}

Note. A dash indicates that the variable was not measured in the study. Discrimination accuracy and response tendency for the episodic recognition memory tasks are given as $d_{\text {ITEM }}^{\prime}$ (for the item recognition task), $d_{A S S O C}^{\prime}$ (for the associative recognition task), $L_{I T E M}$ (for the item recognition task), and $L_{A S S O C}$ (for the associative recognition task). The lower the $L_{I T E M}$ and $L_{A S S O C}$, the more liberal the response tendency (i.e., higher tendency to recognize an item) in the respective episodic recognition task.

in which the recognition validity was high. Nevertheless, as indicated by the SDT analysis of people's use of recognition in inference, old adults' ability to appropriately suspend the recognition heuristic on an item-by-item basis was slightly reduced compared with that of young adults in an environment in which, to make an accurate inference, one often has to go against recognition (i.e., where the recognition validity is low). Furthermore, we obtained tentative support for the hypothesis that these age differences in item adaptivity are mediated by cognitive speed.

Unexpectedly, old adults showed a higher item adaptivity than the young adults in the city environment. Although it has been proposed that old adults might sometimes be more adaptive in their strategy selection than young adults (e.g., Mata et al., 2007), we found no evidence that this difference was due to old adults' higher levels of crystallized knowledge (or age differences in cognitive speed). Therefore, it is currently unclear what brought about old adults' higher item adaptivity in the city environment.

Study 2

Study 2 focuses on the disease environment because, due to differences in recognition validities, the percentage of cases in which it is beneficial to suspend recognition is considerably higher in this than in the city environment ( $40 \%$ vs. $10 \%$, calculated from

Table 4

Intercorrelations Among Cognitive Capacity Measures and Age in Study 1 (and Study 2, Shown in Parentheses)

\begin{tabular}{|c|c|c|c|c|c|c|c|c|c|c|}
\hline Measure & 1 & 2 & 3 & 4 & 5 & 6 & 7 & 8 & 9 & 10 \\
\hline 1. Verbal knowledge & - & $-.35(-.17)$ & $(-.42)$ & $(-.33)$ & $(-.04)$ & $(-.25)$ & (.15) & $-.07(-.03)$ & $-.22(-.14)$ & $.44(.59)$ \\
\hline 2. Digit symbol & & - & $(.62)$ & $(.90)$ & $(.16)$ & $(.36)$ & (.10) & $.28(.39)$ & $.51(.49)$ & $-.80(-.57)$ \\
\hline 3. Identical pictures & & & - & $(.90)$ & $(.07)$ & $(.50)$ & $(.07)$ & $(.38)$ & $(.52)$ & $(-.81)$ \\
\hline 4. Speed composite & & & & - & (.13) & (.47) & (.10) & $(.43)$ & $(.56)$ & $(-.77)$ \\
\hline 5. Flanker & & & & & - & $(.17)$ & $(.38)$ & $(-.06)$ & (.06) & $(-.10)$ \\
\hline 6. Stroop & & & & & & - & (.25) & (.10) & (.31) & $(-.54)$ \\
\hline 7. Inhibition control & & & & & & & & & & \\
\hline composite & & & & & & & - & $(.02)$ & $(.03)$ & $(-.04)$ \\
\hline 8. $d_{\text {ITEM }}^{\prime}$ (episodic & & & & & & & & & & \\
\hline $\begin{array}{l}\text { recognition memory) } \\
\text { 9. } d^{\prime}\end{array}$ & & & & & & & & - & .64 & $-.36(-.37)$ \\
\hline $\begin{array}{l}\text { 9. } d_{A S S O C}^{\prime} \text { (episodic } \\
\text { recognition memory) }\end{array}$ & & & & & & & & & & $-56(-54)$ \\
\hline 10. Age & & & & & & & & & & $\begin{array}{c}-.56(-.54) \\
-\end{array}$ \\
\hline
\end{tabular}

Note. Significance levels are as follows: $r=.22, p=.05$ (Study 1), and $r=.18, p=.05$ (Study 2). Significant correlations are in bold. Discrimination accuracy and response tendency for the episodic recognition memory tasks are given as $d_{\text {ITEM }}^{\prime}$ (for the item recognition task) and $d_{A S S O C}^{\prime}$ (for the associative recognition task). 
the complement of the recognition validities). Accordingly, in the disease environment in Study 1, item adaptivity (as measured by $d_{R H}^{\prime}$ ) was a considerably stronger predictor of overall accuracy in the inference task than in the city environment ( $r \mathrm{~s}=.85$ vs. .48$)$.

In Study 2, we had two goals. The first was to replicate, using a larger sample of participants, the findings in the disease environment in Study 1. As a second goal, in Study 2 we tested whether age differences in item adaptivity were due to cohort differences in incidence knowledge. Specifically, one might argue that these differences could also be due to old adults' outdated disease incidence knowledge rather than cognitive capacities. (Note that using recognition adaptively requires both cognitive capacity and knowledge-for instance, that a particular recognized disease is extinct.) To illustrate, although cholera and leprosy are well recognized, they are currently very rare (yielding a low recognition validity). However, leprosy and cholera were more common in the past, and thus old adults' failure to suspend the recognition heuristic might be due to outdated knowledge about the incidence rate of such diseases. To examine this possibility, we asked participants to also estimate the annual incidence rate for each disease.

\section{Method}

Participants. A total of 60 young adults (33 women, 27 men; mean age $=24.2$ years, range $=19-33$ years $)$ and 60 old adults ( 35 women, 25 men; mean age $=69.7$ years, range $=65-78$ years) participated in Study 2, which was conducted at the Max Planck Institute for Human Development. Young and old adults had about the same number of years of education $(M \mathrm{~s}=16.6 \mathrm{vs}$. 16.0 years). Young and old adults took, on average, around $140 \mathrm{~min}$ and 170 min, respectively, to complete all tasks. The payment scheme was identical to Study 1.

Tasks and materials. In addition to the tasks used in the disease environment condition in Study 1, participants were presented with an estimation task, in which they had to classify each infectious disease into one of the following six frequency categories (indicating their annual incidence rate in Germany): $<1-9$; 10-99; 100-999; 1,000-9,999; 10,000-99,999; and >100,000 (cf. Pachur \& Hertwig, 2006). In addition to the psychometric tests used in Study 1, participants were administered a second measure of processing speed (identical figure task; Lindenberger et al., 1993) and, to investigate the role of inhibitory control in the use of the recognition heuristic, two measures of inhibition control (Stroop task: Stroop, 1935; Eriksen-type flanker task: Eriksen \& Eriksen, 1974; see the online supplemental materials).

Design. The design was a 2 (age group) $\times 2$ (task order) between-subjects design. Within each age group, participants were randomly assigned to one of the two task orders.

Procedure. The instructions and procedure were the same as in the disease environment condition in Study 1, with the exception that after completing the battery of cognitive tests, participants completed the estimation task.

\section{Results}

All analyses of variance included the factors of age group and task order, as well as years of education as a covariate.

Accuracy in the inference task. Table 2 shows that as in Study 1 , old adults were more accurate than young adults, $F(1,110)=$
4.7, $p=.03$. Participants' accuracy on items in which both diseases are recognized (i.e., the knowledge validity) did not differ between the two age groups, $F(1,110)=0.15, p=.70$.

Recognition knowledge. Table 2 shows that, in contrast to Study 1, old adults recognized more diseases than young adults, $F(1,110)=98.1, p=.001$. As a consequence, old adults could apply the recognition heuristic in a lower proportion of items in the inference task than the young adults $(M \mathrm{~s}=0.41$ vs. 0.51), $t(70.1)=7.0, p=.001$. Despite these differences, recognition validities did not differ between young and old adults, $F(1,109)=$ $1.3, p=.26$. As in Study 1, the diseases' recognition rates were highly correlated between young and old adults $(r=.91, p=$ .001). Nevertheless, for some diseases there were marked differences between the age groups, $t(23)=-3.5, p=.002$ (see Table 1). For instance, whereas botulism and trichinosis were recognized by only $7 \%$ and $10 \%$ of the young adults, respectively, $62 \%$ and $72 \%$ of the old adults recognized these diseases (note that there was a similar, though less pronounced, difference also in Study 1 see Table 1).

Item adaptivity. As in Study 1, inferences were considerably faster when they followed rather than contradicted the recognition heuristic. ${ }^{3}$ Although, as in the disease environment in Study 1, old adults again followed recognition in a slightly higher percentage of cases than young adults (see Table 2), this difference was not significant, $F(1,110)=0.77, p=.19$ (one-tailed). An SDT analysis of participants' use of recognition replicated the results of Study 1 . Supporting the reduced item adaptivity hypothesis, old adults showed a lower item adaptivity (as measured by $d_{R H}^{\prime}$ ) than young adults, $F(1,75)=3.0, p=.05 .{ }^{4}$ In contrast to Study 1 , there was a main effect of task order, such that item adaptivity was lower when the recognition task preceded rather than followed the inference task $(M \mathrm{~s}=0.56$ vs. 0.71$), F(1,110)=5.7, p=.02$. (There were additional task order effects, which, however, did not affect our main conclusions; see the Appendix C.) As in Study 1, the interaction between task order and age group was not significant, $F(1,110)=0.50, p=.48$. Young and old adults' tendencies to rely on recognition (as measured by $c_{R H}$ ) did not differ, $F(1$, $110)=0.45, p=.50$.

Cognitive capacity measures. As can be seen in Table 3, the results parallel those of Study 1 (the exception being the response tendency in the item recognition task, which did not differ between young and old adults). Similar to Li et al. (2004), we combined the two measures of inhibitory control into a composite score (see the online supplemental materials). There were not age differences on this composite score or on the flanker task, but old adults showed lower inhibitory control than young adults in the Stroop task. The correlations among the different cognitive capacity measures are shown in Table 4.

\footnotetext{
${ }^{3}$ Young adults' median response time was $1,679 \mathrm{~ms}$ when their inference followed and 2,015.5 ms when it contradicted recognition. For old adults, the median response times were $2,443.5 \mathrm{~ms}$ when the inference followed and 2,754 ms when it contradicted recognition.

${ }^{4}$ Old adults' reduced item adaptivity also affected their accuracy on the items in the inference task in which only one disease was recognized. Here, old adults achieved a lower percentage of accurate inferences than young adults $(M \mathrm{~s}=64.4$ vs. 62.1$), F(1,109)=4.0, p=.05$.
} 
Cognitive capacity and the recognition heuristic. As in Study 1 , verbal knowledge was positively correlated with the number of recognized objects $(r=.53, p=.001)$. A mediation analysis testing the role of cognitive speed in age differences in item adaptivity (now using the composite score of the two speed measures) replicated the results of Study 1 very closely. There were negative associations between item adaptivity (as measured by $\left.d^{\prime}{ }_{R H}\right)$ and age $(\beta=-.18, p=.05)$ and between cognitive speed and age $(\beta=-.77, p=.001)$, and a positive association between $d^{\prime}{ }_{R H}$ and cognitive speed (holding age constant; $\beta=.17, p=.24$; within each age group, the correlation between $d^{\prime}{ }_{R H}$ and speed was $r \mathrm{~s}=-.05$ and .24 , for young and old adults, respectively). The relationship between $d^{\prime}{ }_{R H}$ and age disappeared $(\beta=-.05, p=$ .72), however, when cognitive speed was held constant. In spite of the larger sample size, the very conservative Sobel test was again not significant $(z=0.005, p=.50)$. The effect may thus be small.

Concerning the role of inhibitory control, the scores on the flanker task $(r=.11, p=.22)$, the Stroop task $(r=.04, p=.69)$, and the composite inhibition score $(r=.05, p=.56)$ were at best weakly related to $d^{\prime}{ }_{R H}$. The same applied for the associations with the tendency to rely on recognition (as measured by $c_{R H}$; with $r \mathrm{~s}=$ $-.03,-.02$, and .10 for the flanker, Stroop, and composite scores, respectively).

Estimation task. As described above, one might wonder whether the observed age differences in item adaptivity could be the result of cohort-specific differences in knowledge about the disease incidence rates rather than age differences in cognitive speed. To test this possibility, we calculated for each participant an error index expressing the deviation of the estimated frequency from the actual frequency (e.g., Brown \& Siegler, 1993): ${ }^{5}$

$$
\text { error }=\mid \log (\text { estimate/correct value }) \mid \text {. }
$$

If it is old adults' less accurate frequency knowledge that drives the age differences in item adaptivity (rather than their reduced cognitive speed), these age differences should attenuate when error in the estimation task is held constant. Figure 2 shows old and young adults' estimates for each of the 24 diseases. Although for some diseases, old adults showed a slight tendency to give higher estimates for the rare diseases, young and old adults did not differ in their overall estimation error, $F(1,112)=0.03, p=.87$. Nevertheless, the amount of error was negatively correlated with $d_{R H}^{\prime}(r=-.30, p=.001)$. In other words, participants who were more accurate in the estimation task also used recognition more adaptively on an item-by-item basis in the inference task. Crucially, a mediation analysis revealed that when $d_{R H}^{\prime}$ was regressed on age, holding amount of error in the estimation task constant, the association between age and $d_{R H}^{\prime}$ remained unchanged $(\beta=-.17$ vs. $\beta=-.18$ ). This suggests that age and error in the estimation task had unique (rather than shared) effects on item adaptivity. We thus find no evidence that age differences in participants' incidence knowledge are responsible for the age differences in item adaptivity.

\section{Discussion of Study 2}

Study 2 replicated the main findings of Study 1, thus supporting the idea that the aging decision maker, albeit adaptive between environments, is constrained on an item-by-item basis by agerelated cognitive decline. Nevertheless, there were some unex- pected differences between Studies 1 and 2. First, in Study 2 we could not replicate the age difference in the proportion of choices following recognition (in the disease environment). In particular, young adults' percentage of choices following recognition was almost four percentage points higher than in Study 1 (see Table 2). Comparing the samples of young adults in Studies 1 and 2 directly, however, shows that they did not differ reliably in their proportions of inferences following recognition, $t(78)=-1.13, p=.26$. Second, although young and old adults recognized the same number of diseases in Study 1, old adults recognized more diseases in Study 2 (see Table 2). Interestingly, this difference between Studies 1 and 2 was attenuated when we controlled for differences in verbal knowledge and participants' gender (there was a larger proportion of female participants than in Study 1), $F(1,71)=2.2$, $p=.15$. In sum, our samples of old and young participants differed somewhat between Studies 1 and 2, and some of the differences were associated with differences in the applicability and use of the recognition heuristic. The important point, however, is that even considering these differences, our key findings are robust: Old adults show a reduced ability to use recognition adaptively on an item-by-item basis, and there is some evidence that these age effects are mediated by individual differences in cognitive speed.

Interestingly, Study 2 also demonstrated the interplay between gains and losses in old age. Specifically, old adults could compensate for their deficit in item adaptivity because they recognized a larger number of diseases than young adults and thus could apply their additional factual knowledge about the diseases more frequently (as old adults more often recognized both diseases in the pair comparisons in the inference task than young adults). ${ }^{6}$ As this knowledge had a higher validity than recognition (see Table 2), old adults showed a higher overall accuracy in the inference task than young adults.

\section{General Discussion}

Because of their declining cognitive capacities, old adults may increasingly resort to simple strategies when making decisions. Although the recognition heuristic, like other simple strategies, can sometimes achieve an equal or even higher inferential accuracy than more complex ones (e.g., Gigerenzer \& Goldstein, 1996), the

\footnotetext{
${ }^{5}$ Note that taking the $\log$ of the ratio in Equation 2 corrects for the unequal spacing when the ratio is smaller versus larger than one. The deviations between the estimated and actual frequencies were calculated as follows. First, we identified for each disease the frequency category to which its actual frequency fell. Then, we replaced each of the six frequency categories with the midpoints of each category. For instance, the first category ranging from 1 to 9 was replaced by the value 5 . The last category ">100,000" was replaced by the value 200,000 . These values were then used to calculate the estimation error. Note that the error measure in Equation 2 has the following advantage. It treats two errors-for instance, a response of 5 when the correct value is 500 and a response of 500 when the correct value is 5-equally while being sensitive to the exact values that are involved (e.g., 1,005 and 1,505). Alternative error measures yielded similar results.

${ }^{6}$ To illustrate these associations, when we regressed overall performance in the inference task on item adaptivity, knowledge validity, and the number of recognized objects (controlling for years of education and age), we found that knowledge validity contributed considerably more to overall performance than item adaptivity $(\beta=10.3, p=.001$ vs. $\beta=5.3, p=.001)$.
} 


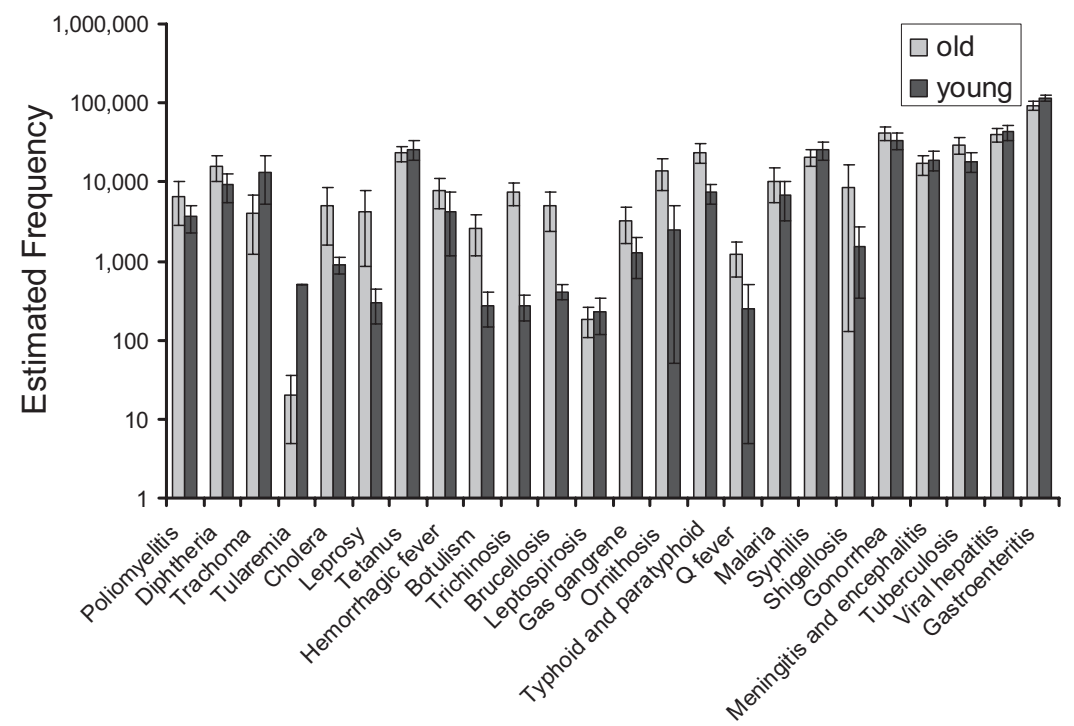

Figure 2. Participants' estimates of the annual incidence rates of the 24 infectious diseases (when they were recognized) in Study 2, separately for young and old adults. Diseases are ordered by actual frequency (starting with the rarest disease). Error bars indicate standard errors of the mean. Note that as there was only one young adult who recognized tularemia, there is no error bar for this disease and age group.

heuristic needs to be used adaptively. Moreover, the heuristic hinges on incomplete knowledge as well as accurate recognition memory. Across two studies, we examined (a) the possibility of age-related changes in the applicability and accuracy of the recognition heuristic and (b) whether old adults show a decreased ability to use the recognition heuristic adaptively (compared with young adults). The main results can be summarized as follows. First, recognition seems to be a robust knowledge base for making inferences across the lifespan: Young and old adults reported having heard of a very similar number of objects, and their average recognition validities were indistinguishable. This result may appear somewhat surprising, as our young and old adults differed considerably with regard to their performances on measures of crystallized and fluid intelligence. Second, both young and old adults followed recognition considerably more often in an environment in which recognition was strongly correlated with the criterion than in an environment in which recognition was only moderately correlated with the criterion. Third, despite this environment adaptivity and levels of knowledge that were (at least) comparable with those of young adults, old adults showed a decreased ability - in an environment with a low recognition validity - to selectively suspend the use of the recognition heuristic when it led to an incorrect inference. We also found some support for the hypothesis that this age-related deficit in item adaptivity is mediated by cognitive speed. Unexpectedly, in the high recognition validity environment, old adults showed a higher item adaptivity. In what follows, we discuss the implications of our results.

\section{The Recognition Heuristic and Episodic Recognition Memory}

What do our results indicate concerning the contribution of episodic recognition memory in the use and usefulness of recognition in decision making? Despite old adults' lower performance on measures of episodic recognition memory, the two age groups differed only slightly in terms of their recognition knowledge (i.e., which and how many cities and diseases they recognized). Moreover, Table 5 shows the partial correlations between measures of episodic memory and the number of objects recognized in Studies 1 and 2 (controlling for verbal knowledge). Across both studies, it appears as though there is a tendency for people to recognize more objects (i.e., cities, diseases) the lower their discrimination ability and the more liberal their tendency to recognize a word in the episodic recognition tasks. The correlations, however, are generally low and slightly higher only for measures of associative episodic recognition memory. The recognition knowledge tapped by the recognition heuristic may thus be relatively unaffected by individual differences in episodic memory.

Table 5

Partial Correlation (Controlling for Verbal Knowledge) Between Measures of Episodic Recognition Memory and the Number of Recognized Objects in Study 1 (Results for Study 2 Are Shown in Parentheses)

\begin{tabular}{llllll}
\hline & \multicolumn{4}{c}{ Type of episodic recognition } \\
\cline { 2 - 3 } \cline { 5 - 6 } Environment & \multicolumn{2}{c}{ Item recognition } & & \multicolumn{2}{c}{ Associative recognition } \\
\cline { 2 - 3 } \cline { 5 - 6 } Infectious diseases & $-.11(-.16)$ & $-.16(.13)$ & $-.14(-.21)$ & $-.24(-.17)$ \\
Cities & -.13 & .03 & -.04 & -.14 \\
\hline
\end{tabular}

Note. Significance levels are as follows: $r=.31, p=.05$ (Study 1), and $r=.18, p=.05$ (Study 2). Discrimination accuracy and response tendency for the episodic recognition memory tasks are given as $d_{\text {ITEM }}^{\prime}$ (for the item recognition task), $d_{A S S O C}^{\prime}$ (for the associative recognition task), $L_{I T E M}$ (for the item recognition task), and $L_{A S S O C}$ (for the associative recognition task). 
On the other hand, consider participants' responses in the inference task, in which each object was presented 23 times. If participants did not take into consideration any episodic knowledge in this task, previously recognized and unrecognized objects would become increasingly indistinguishable over the course of the experiment. There was indeed a tendency for the percentage of inferences following recognition to decrease from the first to the second half of the trials (Study 1: $M \mathrm{~s}=81.8 \%$ vs. $80.9 \%$; Study $2: M \mathrm{~s}=70.4 \%$ vs. $68.8 \%)$. However, the effect was smallStudy 1: $F(1,75)=1.14, p=.29$; Study $2: F(1,117)=3.2, p=$ .08 - and of the same size for both age groups-Study 1: $F(1$, $75)=0.80, p=.37$; Study $2: F(1,117)=0.53, p=.47$.

So at least when the presentation of the novel names is embedded in the inference task, people seem to recruit episodic recognition memory, helping them to correctly attribute the increased familiarity to the experimental presentation. Note the similarity to findings on the false fame effect (i.e., using experimentally induced familiarity for inferences about the world outside the lab; Jacoby, Kelley, Brown, \& Jasechko, 1989). The false fame effect is considerably reduced when inference follows presentation immediately (rather than with a 24-hr delay; see Jacoby et al., 1989) or when participants are reminded during inference of the possibility that the familiarity with a word might be experimentally induced (Multhaup, 1995).

\section{Gains and Losses in Decision-Making Abilities in Old Age}

Our results closely match those of Mata et al. (2007) by showing that both young and old adults are able to adjust their decision processes as a function of environment structure. Nevertheless, both our and Mata et al.'s results suggest that old adults' limited cognitive abilities can lead them to rely on simpler decision strategies regardless of whether the environment favors these. In the present studies, old adults more often relied on recognition even on items for which this was not an appropriate strategy.

Our results are also relevant for the discussion of whether the negative impact of cognitive decline on decision making may be offset by higher levels of knowledge (Hanoch, Wood, \& Rice, 2007; Mather, 2006; Tentori, Osherson, Hasher, \& May, 2001). We are not aware of studies that have directly tested this claim using real-world domains in which inferences can be evaluated on the basis of objective criteria of accuracy (cf. Thornton \& Dumke, 2005). The results of our studies, which tapped participants' realworld knowledge (about existing cities and diseases), suggest that in spite of their reduced item adaptivity, older adults can sometimes achieve similar or even superior overall levels of inference performance than young adults because of greater (recognition) knowledge. Because they recognized more objects (see Table 2), old adults more often recognized both objects in the inference task; in those cases the recognition heuristic is not applicable, and old adults' reduced item adaptivity did not come into play.

\section{Conclusions}

Although a simple strategy_ and thus an attractive tool for making inferences with limited cognitive resources - the recognition heuristic can be negatively affected by too much knowledge, inaccurate recognition memory, or deficits in its adaptive use. Our results suggest that old adults' greater experience and deficits in episodic recognition memory do not hamper the inferential power of the heuristic. Moreover, although old adults applied the strategy somewhat less successfully on an item-by-item basis, they were overall adaptive by relying more often on recognition when its validity was high compared with when it was low. In other words, our results further document that aging decision makers are adaptive and have at their disposal a toolbox of strategies, such as the recognition heuristic, that are robust in the face of cognitive decline.

\section{References}

Allen, P. A., Smith, A. F., Groth, K. E., Pickle, J., Grabbe, J. W., \& Madden, D. J. (2002). Differential age effects for case and hue mixing in visual word recognition. Psychology and Aging, 17, 622-635.

Baltes, P. B., Staudinger, U. M., \& Lindenberger, U. (1999). Lifespan psychology: Theory and application to intellectual functioning. Annual Review of Psychology, 50, 471-507.

Banks, W. P. (1970). Signal detection theory and human memory. Psychological Bulletin, 74, 81-99.

Baron, R. M., \& Kenny, D. A. (1986). The moderator-mediator variable distinction in social psychological research: Conceptual, strategic, and statistical considerations. Journal of Personality and Social Psychology, 51, 1173-1182

Bröder, A., \& Schiffer, S. (2003). Take the best versus simultaneous feature matching: Probabilistic inferences from memory and effects of representation format. Journal of Experimental Psychology: General, 132, 277-293.

Bröder, A., \& Schiffer, S. (2006). Stimulus format and working memory in fast and frugal strategy selection. Journal of Behavioral Decision Making, 19, 361-380.

Brown, N. R., \& Siegler, R. S. (1993). Metrics and mappings: A framework for understanding real-world quantitative estimation. Psychological Review, 100, 511-534.

Chasseigne, G., Mullet, E., \& Stewart, T. R. (1997). Aging and multiple cue probability learning: The case of inverse relationships. Acta Psychologica, 97, 235-252.

Chen, Y., \& Sun, Y. (2003). Age differences in financial decisions-making: Using simple heuristics. Educational Gerontology, 29, 627-635.

Craik, F. I. M., \& McDowd, J. M. (1987). Age differences in recall and recognition. Journal of Experimental Psychology: Learning, Memory, and Cognition, 13, 474-479.

Dunlosky, J., \& Hertzog, C. (2000). Updating knowledge about strategy effectiveness: A componential analysis of learning about strategy effectiveness from task experience. Psychology and Aging, 15, 462-474.

Eriksen, B. A., \& Eriksen, C. W. (1974). Effects of noise letters upon the identification of a target letter in a nonsearch task. Perception and Psychophysics, 16, 143-149.

Finucane, M. L., Mertz, C. K., Slovic, P., \& Schmidt, E. S. (2005). Task complexity and older adults' decision-making competence. Psychology and Aging, 20, 71-84.

Frosch, C., Beaman, C. P., \& McCloy, R. (2007). A little learning is a dangerous thing: An experimental demonstration of ignorance-driven inference. Quarterly Journal of Experimental Psychology, 60, 1329_ 1336.

Gigerenzer, G., \& Goldstein, D. G. (1996). Reasoning the fast and frugal way: Models of bounded rationality. Psychological Review, 103, 650-669.

Gigerenzer, G., \& Selten, R. (Eds.). (2001). Bounded rationality: The adaptive toolbox. Cambridge, MA: MIT Press.

Gigerenzer, G., Todd, P. M., \& the ABC Research Group. (1999). Simple heuristics that make us smart. New York, NY: Oxford University Press.

Goldstein, D. G., \& Gigerenzer, G. (2002). Models of ecological rationality: The recognition heuristic. Psychological Review, 109, 75-90.

Gusnard, D. A., Akbudak, E., Shulman, G. L., \& Raichle, M. E. (2001). Medial prefrontal cortex and self-referential mental activity: Relation to 
a default mode of brain function. Proceedings of the National Academy of Sciences, USA, 98, 4259-4264.

Hanoch, Y., Wood, S., \& Rice, T. (2007). Bounded rationality, emotions and older adult decision making: Not so fast and yet so frugal. Human Development, 50, 333-358.

Hasher, L., \& Zacks, R. T. (1988). Working memory, comprehension, and aging: A review and a new view. In G. H. Bower (Ed.), The psychology of learning and motivation (Vol. 22, pp. 193-225). New York, NY: Academic Press.

Healy, M. R., Light, L. L., \& Chung, C. (2005). Dual-process models of associative recognition in young and older adults: Evidence from receiver operating characteristics. Journal of Experimental Psychology: Learning, Memory, and Cognition, 31, 768-788.

Hertwig, R., Pachur, T., \& Kurzenhäuser, S. (2005). Judgments of risk frequencies: Tests of possible cognitive mechanisms. Journal of Experimental Psychology: Learning, Memory, and Cognition, 31, 621-642.

Hess, T. M., McGee, K. A., Woodburn, S. M., \& Bolstad, C. A. (1998). Age-related priming effects in social judgments. Psychology and Aging, 13, 127-137.

Hilbig, B. E., \& Pohl, R. F. (2008). Recognizing users of the recognition heuristic. Experimental Psychology, 55, 394-401.

Jacoby, L. L., Kelley, C., Brown, J., \& Jasechko, J. (1989). Becoming famous overnight: Limits on the ability to avoid unconscious influences of the past. Journal of Personality and Social Psychology, 56, 326-338.

Judd, C. M., \& Kenny, D. A. (1981). Process analysis: Estimating mediation in treatment evaluations. Evaluation Review, 5, 602-619.

Kelley, R., \& Wixted, J. T. (2001). On the nature of associative information in recognition memory. Journal of Experimental Psychology: Learning, Memory, and Cognition, 20, 701-722.

Kliegl, R., \& Lindenberger, U. (1993). Modeling intrusions and correct recall in episodic memory: Adult age differences in encoding of list context. Journal of Experimental Psychology: Learning, Memory, and Cognition, 19, 617-637.

La Voie, D., \& Light, L. L. (1994). Adult age differences in repetition priming: A meta-analysis. Psychology and Aging, 9, 539-553.

Lemaire, P., Arnaud, L., \& Lecacheur, M. (2004). Adults' age-related differences in adaptivity of strategy choices: Evidence from computational estimation. Psychology and Aging, 10, 467-481.

Li, S.-C., Lindenberger, U., Hommel, B., Aschersleben, G., Prinz, W., \& Baltes, P. B. (2004). Transformations in the couplings among intellectual abilities and constituent cognitive processes across the life span. Psychological Science, 15, 155-163.

Lindenberger, U., Mayr, U., \& Kliegl, R. (1993). Speed and intelligence in old age. Psychology and Aging, 8, 207-220.

MacKinnon, D. P., Warsi, G., \& Dwyer, J. H. (1995). A simulation study of mediated effect measures. Multivariate Behavioral Research, 30, 41-62.

Macmillan, N. A. (1993). Signal detection theory as data analysis method and psychological decision model. In G. Keren \& C. Lewis (Eds.), Methodological and quantitative issues in the analysis of psychological data (Vol. 1, pp. 21-57). Hillsdale, NJ: Erlbaum.

Macmillan, N. A., \& Creelman, C. D. (1990). Detection theory measures of response bias. Psychological Bulletin, 107, 401-413.

Mata, R., Schooler, L. J., \& Rieskamp, J. (2007). The aging decision maker: Cognitive aging and the adaptive selection of decision strategies. Psychology and Aging, 22, 796-810.

Mather, M. (2006). A review of decision making processes: Weighing the risks and benefits of aging. In L. L. Carstensen \& C. R. Hartel (Eds.), When I'm 64: Committee on Aging Frontiers in Social Psychology, Personality, and Adult Developmental Psychology (pp. 145-173) Washington, DC: National Academies Press.

Multhaup, K. S. (1995). Aging, source, and decision criteria: When false fame errors do and do not occur. Psychology and Aging, 10, 492-497.

Mutter, S. A., Strain, L. M., \& Plumlee, L. F. (2007). The role of age and prior belief in contingency judgment. Memory \& Cognition, 35, 875-884.
Naveh-Benjamin, M. (2000). Adult-age differences in memory performance: Tests of an associative deficit hypothesis. Journal of Experimental Psychology: Learning, Memory, and Cognition, 26, 1170-1187.

Neely, J. H., \& Payne, D. G. (1983). A direct comparison of recognition failure rates for recallable names in episodic and semantic memory tests. Memory \& Cognition, 11, 161-171.

Oppenheimer, D. M. (2003). Not so fast! (and not so frugal!): Rethinking the recognition heuristic. Cognition, 90, B1-B9.

Oppenheimer, D. M. (2004). Spontaneous discounting of availability in frequency judgment tasks. Psychological Science, 15, 100-105.

Pachur, T., \& Biele, G. (2007). Forecasting from ignorance: The use and usefulness of recognition in lay predictions of sports events. Acta Psychologica, 125, 99-116.

Pachur, T., Bröder, A., \& Marewski, J. M. (2008). The recognition heuristic in memory-based inference: Is recognition a non-compensatory cue? Journal of Behavioral Decision Making, 21, 183-210.

Pachur, T., \& Hertwig, R. (2006). On the psychology of the recognition heuristic: Retrieval primacy as a key determinant of its use. Journal of Experimental Psychology: Learning, Memory, and Cognition, 32, 9831002.

Pachur, T., Todd, P. M., Gigerenzer, G., Schooler, L. J., \& Goldstein, D. G. (in press). When is ignorance an adaptive tool? In P. M. Todd, G. Gigerenzer, \& the ABC Research Group (Eds.), Ecological rationality: Intelligence in the world. New York, NY: Oxford University Press.

Payne, J. W., Bettman, J. R., \& Johnson, E. J. (1993). The adaptive decision maker. New York, NY: Cambridge University Press.

Pleskac, T. J. (2007). A signal detection analysis of the recognition heuristic. Psychonomic Bulletin and Review, 14, 379-391.

Pohl, R. (2006). Empirical tests or the recognition heuristic. Journal of Behavioral Decision Making, 19, 251-271.

Ratcliff, R., Thapar, A., \& McKoon, G. (2007). Application of the diffusion model to two-choice tasks for adults 75-90 years old. Psychology and Aging, 22, 56-66.

Rieskamp, J., \& Hoffrage, U. (2008). Inferences under time pressure: How opportunity costs affect strategy selection. Acta Psychologica, 127, 258-276.

Sanford, A. J., \& Maule, A. J. (1971). Age and the distribution of observing responses. Psychonomic Science, 23, 419-420.

Scheibehenne, B., \& Bröder, A. (2007). Predicting Wimbledon tennis results 2005 by mere player name recognition. International Journal of Forecasting, 23, 415-426.

Schooler, L., \& Hertwig, R. (2005). How forgetting aids heuristic inference. Psychological Review, 112, 610-628.

Shing, Y. L., Werkle-Bergner, M., Li, S.-C., \& Lindenberger, U. (2008). Associative and strategic components of episodic memory: A life-span dissociation. Journal of Experimental Psychology: General, 137, 495-513.

Snodgrass, J. G., \& Corwin, J. (1988). Pragmatics of measuring recognition memory: Applications to dementia and amnesia. Journal of Experimental Psychology: General, 117, 34-50.

Snook, B., \& Cullen, R. M. (2006). Recognizing national hockey league greatness with an ignorance-based heuristic. Canadian Journal of Experimental Psychology, 60, 33-43.

Sobel, M. E. (1982). Asymptotic confidence intervals for indirect effects in structural equation models. In S. Leinhardt (Ed.), Sociological methodology (pp. 290-312). Washington, DC: American Sociological Association.

Spencer, W. D., \& Raz, N. (1995). Differential effects of aging on memory for content and context: A meta-analysis. Psychology and Aging, 10, $527-539$.

Stanislaw, H., \& Todorov, N. (1999). Calculation of signal detection theory measures. Behavior Research Methods, Instruments, \& Computers, 31 , $137-149$.

Stroop, J. R. (1935). Studies of interference in serial verbal reactions. Journal of Experimental Psychology, 18, 643-661.

Tentori, K., Osherson, D., Hasher, L., \& May, C. (2001). Wisdom and 
aging: Irrational preferences in college students but not older adults. Cognition, 81, B87-B96.

Thornton, W. J. L., \& Dumke, H. A. (2005). Age differences in everyday problem-solving and decision-making effectiveness: A meta-analytic review. Psychology and Aging, 20, 85-99.

Toth, J. P., \& Daniels, K. A. (2002). Effects of prior experience on judgments of normative word frequency: Automatic bias and correction. Journal of Memory and Language, 46, 845-874.

Tulving, E. (1972). Episodic and semantic memory. In E. Tulving \& W.
Donaldson (Eds.), Organization of memory (pp. 381-403). New York, NY: Academic Press.

Volz, K. G., Schooler, L. J., Schubotz, R. I., Raab, M., Gigerenzer, G., \& von Cramon, D. Y. (2006). Why you think Milan is larger than Modena: Neural correlates of the recognition heuristic. Journal of Cognitive Neuroscience, 18, 1924-1936.

Yonelinas, A. (1997). Recognition memory ROCs for item and associative information: The contribution of recollection and familiarity. Memory \& Cognition, 25, 747-763.

\section{Appendix A}

\section{Calculation of Signal Detection Theory Parameters for the Use of Recognition in the Inference Task}

Both signal detection theory parameters, $d_{R H}^{\prime}$ and $c_{R H}$, are estimated on the basis of each participant's hit and false alarm rates. The hit rate was defined as the proportion of cases in which, when a person was presented with an item in which the recognized object had a higher criterion value than the unrecognized object, his or her inference followed recognition:

$$
\left.H=\frac{\left(n_{\text {inference follows recognition when } R H=\text { correct }}+0.5\right)}{\left(n_{R H}=\right.\text { correct }}+1\right) \text {. }
$$

The false alarm rate was defined as the proportion of cases in which, when a person was presented with an item in which the recognized object had a lower criterion value than the unrecognized object, his or her inference followed recognition:

$$
\left.F A=\frac{\left(n_{\text {inference follows recognition when } R H=\text { incorrect }}+0.5\right)}{\left(n_{R H}=\right.\text { incorrect }}+1\right) .
$$

(The purpose of transforming the hit rate and the false alarm rate by adding 0.5 in the nominator and 1 in the denominator was to avoid problems when the untransformed hit or false alarm rates equal 1 or 0; Snodgrass \& Corwin, 1988.) On the basis of the hit and the false alarm rates, $d_{R H}^{\prime}$ was calculated for each participant as $d_{R H}^{\prime}=z(H)-z(F A)$, where $z(H)$ is the $z$ value (in the standard normal distribution) that cuts off $H$ of the distribution, and $z(F A)$ is the $z$ value (in the standard normal distribution) that cuts off $F A$ of the distribution. We estimated $c_{R H}$ as the average $z$ values of $H$ and $F A$, multiplied by negative one (Macmillan, 1993):

$$
c_{R H}=-\frac{z(H)+z(F A)}{2} .
$$

\section{Appendix B}

\section{Mediation Analyses for the High Recognition Validity Environment}

To examine the possible role of (a) speed, (b) verbal knowledge, and (c) knowledge validity in the age differences in item adaptivity in the city environment (in which the recognition validity was high), we conducted three mediation analyses. Note that knowledge validity is supposed to measure the amount of relevant factual knowledge about the inference domain (i.e., American cities). Interestingly, knowledge validity and verbal knowledge were uncorrelated $(r=-.03, p=.84)$.

Speed. There was a positive association between age and $d_{R H}^{\prime}$ ( $\beta=.46, p=.003)$, a negative association between cognitive speed and age $(\beta=-.86, p=.001)$, and a weak negative association between cognitive speed and $d_{R H}^{\prime}$, holding age constant ( $\beta=-.26, p=.11)$. Finally, holding cognitive speed constant, the positive association between age and $d_{R H}^{\prime}$ held up $(\beta=.90, p=$ $.002)$, suggesting that cognitive speed does not mediate the age differences in $d_{R H}^{\prime}$ in the high recognition validity environment.
Verbal knowledge. There was no association between item adaptivity and verbal knowledge, keeping age constant $(\beta=.08$, $p=.63)$, and accordingly, the association between $d_{R H}^{\prime}$ and age was unaffected by whether knowledge was held constant $(\beta=.45$, $p=.004$ vs. $\beta=.43, p=.01)$. Knowledge had thus no mediating effect on the association between age and $d_{R H}^{\prime}$ in the high recognition validity environment.

Knowledge validity. In addition to the positive association between age and $d_{R H}^{\prime}$ (see above), there was a weak negative association between knowledge validity and age $(\beta=-.10, p=$ $.53)$, and a positive association between knowledge validity and $d_{R H}^{\prime}$, holding age constant $(\beta=.22, p=.13)$. Finally, holding knowledge validity constant, the positive association between age and $d_{R H}^{\prime}$ remained practically unchanged $(\beta=.48, p=.002)$, suggesting that knowledge validity does not mediate the age differences in $d_{R H}^{\prime}$ in the high recognition validity environment. 


\section{Appendix C}

\section{Task-Order Effects in Study 2}

Recognition knowledge. There was a main effect of task order on the number of recognized diseases, $F(1,110)=6.2, p=.014$ (see Table 2). Contrary to the possibility that the inference task induces false recognition, however, the number of recognized diseases was lower when the inference task preceded, compared with when it followed, the recognition task. One possible explanation for this result is that participants were more cautious in the recognition task after they had been presented with the diseases repeatedly during the inference task. Given that the effect of task order occurred neither in Study 1 nor in previous studies of the recognition heuristic (Pachur et al., 2008; Pachur \& Hertwig, 2006; Pohl, 2006), we treat this result with caution. The interaction between task and age group was not significant, $F(1,110)=2.5$, $p=.12$

In addition, there was a main effect of task order on recognition validity, with a lower recognition validity when the recognition task preceded compared with when it followed the inference task $(M \mathrm{~s}=0.59$ vs. 0.61$), F(1,109)=5.7, p=.02$. The size of this effect was the same for young and old adults, $F(1,109)=0.01$, $p=.92$. Note that in Study 1, the difference between the recognition validities in the two task-order conditions was the same$M \mathrm{~s}=0.60$ and 0.62 - when the inference task preceded and followed, respectively, the recognition task. Because of the small sample size, however, this difference had not been significant, $F(1$, 36) $=0.88, p=.35$

Use of recognition. Task order did not affect the proportion of inferences following recognition overall, $F(1,110)=0.49, p=$ .49 , but there was a significant interaction between task order and age group, $F(1,110)=9.1, p=.003$. Specifically, the proportion of inferences following the recognition heuristic was lower for young adults but higher for old adults when the recognition task followed rather than preceded the inference task. Similarly-as in Study 1 -there was an interaction between age group and task order, $F(1,110)=8.0, p=.006$, on the tendency to rely on recognition in the inference task (as measured by $c_{R H}$ ). Specifically, young adults were more conservative in their reliance on recognition in the inference task when the task followed, compared with when it preceded, the recognition task $(M \mathrm{~s}=-0.42$ vs. $-0.58)$. Old adults, by contrast, showed the opposite pattern and were more liberal in their reliance on recognition when the inference task followed, compared with when it preceded, the recognition task $(M \mathrm{~s}=-0.72$ vs. -0.42$)$.

Received June 24, 2008 Revision received April 15, 2009

Accepted July 13, 2009 